FORMATION Formation emploi

Revue française de sciences sociales

138 | Avril-Juin 2017

La professionnalisation dans l'enseignement supérieur : formes et effets variés

\title{
Fabriquer / devenir un enseignant du supérieur professionnalisant. Le tutorat en IUT
}

How higher education teachers learn how to follow students performances in companies during internships? A study of the tutoring organization and its socializations' effects

Wie Fabriziert / wird man Lehrer im professionalisierenden Höheren

Bildungswesen? Tutorium im IUT

Fabricar / volverse docente de nivel superior profesionalizante. Los tutores en IUT

\section{Stéphanie Tralongo}

\section{OpenEdition}

Journals

\section{Édition électronique}

URL : https://journals.openedition.org/formationemploi/5103

DOI : $10.4000 /$ formationemploi.5103

ISSN : 2107-0946

Éditeur

La Documentation française

Édition imprimée

Date de publication : 17 juillet 2017

Pagination : 165-183

ISSN : 0759-6340

Référence électronique

Stéphanie Tralongo, «Fabriquer / devenir un enseignant du supérieur professionnalisant. Le tutorat en IUT », Formation emploi [En ligne], 138 | Avril-Juin 2017, mis en ligne le 10 juillet 2019, consulté le 21 septembre 2021. URL : http://journals.openedition.org/formationemploi/5103 ; DOI : https://doi.org/ 10.4000/formationemploi.5103 


\title{
Fabriquer / devenir un enseignant du supérieur professionnalisant. Le tutorat en IUT
}

\author{
StéPHANie Tralongo \\ Maîtresse de conférences en sociologie. Equipe MEPS (Modes Espaces et Processus de \\ Socialisation) du Centre Max Weber, UMR 5283
}

Résumé

Fabriquer / devenir un enseignant du supérieur professionnalisant. Une enquête en IUT sur le tutorat

Comment les enseignants et enseignants-chercheurs en Instituts universitaires de technologie (IUT) apprennent-ils à participer à des activités de professionnalisation des étudiants ? L'exemple choisi est un dispositif de tutorat, qui signifie un suivi régulier et répété d'étudiants en entreprise par les personnels d'enseignement et de recherche. L'enquête monographique met en évidence un processus de socialisation s'appuyant sur des dispositions plus ou moins préalablement constituées dans différents contextes (familiaux, d'études, professionnels), qui contribue à façonner une hétérogénéité dans les pratiques professionnelles et des relations resserrées avec le monde économique.

Mots clés : tutorat, enseignement supérieur, IUT - Institut universitaire de technologie, enseignant, socialisation professionnelle, activité professionnelle, représentation du travail

Abstract

How higher education teachers learn how to follow students performances in companies during internships? A study of the tutoring organization and its socializations'effects

The article focuses on the ways in which teachers in higher education learn how to participate in the professional training of students. It is based on a sociology of socialization theoretical approach. The issue deals with the question of the organization of relationships between a University Institute of Technology (IUT) and the workplace. The example developed by a monographic method is tutoring : on our field of study it means teachers supporting students on the workplace during internships. The article studies the institutional organization of tutoring and its appropriation by teachers.

Keywords: tutoring, higher education, university institute of technology, teacher, professional socialisation, vocational activity, perception of work

Journal of Economic Literature: I 23, M 54

Traduction : Auteure. 
La professionnalisation des étudiants, des formations, des savoirs, de l'université étant polymorphe, polysémique ${ }^{1}$, ses modalités de mises en œuvre et ses effets posent d'autant plus question. On se propose de s'intéresser à ceux par lesquels elle est censée passer - autrement dit les enseignants et enseignants-chercheurs - en se demandant de quelles manières ils sont amenés à participer à cette professionnalisation. La recherche est restreinte au terrain des Instituts universitaires de technologie (IUT).

Créés au milieu des années soixante, les IUT concentrent, au sein des universités, des pratiques de professionnalisation des étudiants qui restent largement à mettre au jour et à interroger. Cent-treize IUT sont ainsi répartis sur tout le territoire français. Ils délivrent principalement deux types de diplômes : l'un en propre, le Diplôme universitaire de technologie (DUT) et un autre, partagé avec d'autres composantes universitaires, la licence professionnelle (Maillard et Veneau, 2006), comptabilisant entre 4 et $5 \%$ des effectifs étudiants ${ }^{2}$. En termes d'effectifs enseignant, pour l'année 2014-2015, les IUT comptaient 9384 titulaires d'enseignement et de recherche, se répartissant comme suit : $56 \%$ d'enseignants-chercheurs (professeurs et maîtres de conférences) et $47 \%$ d'enseignants du second degré détachés dans le supérieur (Certificat d'aptitude au professorat de l'enseignement et agrégation) ${ }^{3}$.

Au regard des types de diplômes de ces titulaires, l'hypothèse est celle une double coupure a priori : d'une part, on ne peut présupposer qu'ils ont été massivement familiarisés aux formations en IUT pendant leur propre parcours d'étudiant et, d'autre part, dans leur pratique professionnelle, ils auront à former majoritairement des étudiants pour des métiers ou univers professionnels différents des leurs.

Dès lors, quels sont les attendus institutionnels concernant les activités de professionnalisation des étudiants auxquels les enseignants doivent prendre part ? Comment en prennent-ils connaissance et que font-ils ? S'agit-il pour les enseignants de transformer leurs pratiques professionnelles ? Si c'est le cas, comment la transformation s'organiset-elle ? Comment est-elle prise en charge institutionnellement et avec quels effets ?

La problématique générale de la recherche s’inscrit ainsi dans une sociologie de la socialisation et, dans ce cas, de la socialisation professionnelle (Hugues, 1996 ; Dubar, 1991) exercée au cours de l'activité. La piste suivie ici est celle des effets de l'organisation institutionnelle des relations aux entreprises pour les enseignants titulaires. L'entrée s'opère à partir d'une activité particulière, le "suivi ${ }^{4}$ » d'étudiants en entre-

1. Voir, par exemple, Quenson et Coursaget, 2012 ; Demazière, 2009 ; Maillard, 2012 ; Maillard \& Veneau, 2006 ; Stavrou, 2011.

2. Source : MESR-DEGESIP/DGRI-SIES, note d'information 14.01 (2014).

3.Source : RERS 2015 (Repères et Références Statistiques, ministère de l'Education nationale, (www.education. gouv.fr/cid57096/reperes-et-references-statistiques.html).

4. Il s'agit du terme indigène. Une présentation plus précise du tutorat est effectuée en début de troisième partie. Il est à noter qu'il s'agit d'une autre forme d'organisation et d'autres acteurs que ceux étudiés sur divers 
prise, intitulé tutorat. Sur le terrain d'un IUT étudié de façon monographique (voir l'encadré méthodologique 1, en fin d'introduction), il consiste à affecter à chaque alternant ${ }^{5}$ un référent travaillant dans l'IUT, chargé de "suivre " son immersion en entreprise (au moyen de rencontres ponctuelles effectuées en entreprise).

L'intérêt pour cette activité vient de son importance pour l'institution ${ }^{6}$ : elle doit concerner tous les personnels titulaires d'enseignement et de recherche dans cet IUT et, au-delà, certains personnels administratifs et vacataires. Le but explicite est une acculturation au monde de l'entreprise ; cela se matérialise par un effectif conséquent de tuteurs. Sur l'année de l'enquête (2012 -2013), six-cents alternants ont occupé un poste en entreprise, pour environ cent-quarante tuteurs (un tuteur pouvant "suivre " plusieurs alternants). Parmi cet effectif, peu fluctuant chaque année, on note la présence de novices diversement familiarisés aux pratiques de cet IUT.

Les questions que se pose l'institution portent alors à la fois sur la transmission (fabrication de nouveaux tuteurs) et sur l'harmonisation des pratiques (entre les anciens et nouveaux tuteurs, entre les tuteurs, entre les diplômes).

L'importance institutionnelle du tutorat a pour conséquence une production abondante d'écrits, de formations, de pratiques, d'instruments et d'outils qui ont ainsi été élaborés au fil des ans pour l'organiser, offrant la possibilité d'étudier le travail de cadrage produit par l'IUT sur cette activité.

Le terrain, ainsi que les éléments théoriques mobilisés pour évoquer la question de socialisation, dans ce contexte, sont présentés dans la première partie de l'article.

Il est à souligner que ce terrain possède plusieurs spécificités par rapport aux autres IUT. Elles concernent, d'une part, la conception centrale de l'objectif de professionnalisation des étudiants qui est étendue à tous les diplômes et, d'autre part, le travail de mise en cohérence entre cette vision politique et les dispositifs, instruments, outils pédagogiques et managériaux développés pour l’opérationnaliser. Cela invite à considérer ce terrain non comme représentatif des pratiques professionnalistes en IUT, mais

terrains, tels Bourdon \& ali. (2014) ou Allouch et Van Zanten, mais dont le point commun réside dans le fait qu' on observe "une remise en cause à la fois de la distance professorale et de la centration des enseignants sur les seuls apprentissages de type intellectuel " (Allouch \& Van Zanten, 2008).

5. Le terme d'alternant, générique, est utilisé ici parce que les statuts juridiques de ceux qui forment les promotions dans l'IUT étudié sont différents (étudiant, apprenti, stagiaire de la formation continue).

6. Précisons que le choix de focale sur une activité de professionnalisation constitue une coupure artificielle dans un ensemble de pratiques qui s'avèrent, sur le terrain, particulièrement nombreuses et diversifiées, et dont le nombre a un effet cumulatif contribuant à produire un cadre socialisateur pour les enquêtés. Les propos des enquêtés permettent d'observer un enchevêtrement et une articulation des effets des dispositifs, qui sont mobilisés dans l'analyse. 
plutôt comme l'illustration d'une représentation (particulièrement organisée et à visée homogénéisante) de la professionnalisation des étudiants.

La seconde partie est consacrée à l'analyse du tutorat et des différentes manières dont les enquêtés ont débuté et cheminé dans cette activité.

\section{Encadré 1 : Méthodologie}

La méthodologie de production des données relève ici d'une variété de techniques, organisée à partir d'une ethnographie. Celle-ci a été menée dans différents contextes liés aux IUT (Institut universitaire de technologie), approchés par l'occupation de plusieurs fonctions pendant une douzaine d'année de direction (en tant que chef de département, responsable de licences professionnelles, et directrice adjointe d'un IUT). À partir de 2012, un travail d'observation et de « participation observante » (Alam \& al. (2012) a été réalisé sur des situations et dispositifs de professionnalisation des étudiants et les dispositifs de management des personnels d'un IUT.

Un corpus de documents qualitatifs (tels que notes internes, dossiers d'évaluation des diplômes, dispositifs managériaux, matériel pédagogique...) et quantitatifs (issus du service de statistique décisionnelle) a été constitué. Enfin, un à deux entretiens auprès de vingt-six titulaires enseignants et enseignants-chercheurs (sur trente-deux), de deux heures en moyenne, se sont déroulés à partir de 2013, après la cessation de toute responsabilité administrative.

Les thèmes d'entretien concernaient : 1 ) les choix d'orientation au moment du baccalauréat et dans les études supérieures, les parcours et établissement de formation ; les modalités pédagogiques vécues en tant qu'étudiant ; 2 ) les premières expériences et le passé professionnel de l'enquêté ; 3) les manières d'avoir pris connaissance de l'existence des IUT, et l'histoire du recrutement ; 4) les variations dans la carrière au sein de I'IUT étudié (dont les prises de responsabilités administratives), la liste des cours administrés et la variation dans les appétences envers ceux-ci au cours du temps ; 5) les origines sociales, niveaux d'études, professions et rapport au monde professionnel de la parentèle ; 6 ) les perspectives professionnelles à moyen terme.

Le tableau 1 récapitule différentes informations pour les vingt-six enquêtés : les pôles disciplinaires, corps d'Etat, sexe, parcours de formation, origines sociales à partir de la profession du père et ancienneté (tenant compte des éventuelles périodes de vacation). 
Tableau 1 : Présentation des enquêtés selon les pôles disciplinaires, corps d'Etat, sexe, cursus et PCS du père

\begin{tabular}{|l|l|}
\hline Pôles disciplinaires & 5 en lettres, langues, sciences humaines et sociales \\
& 12 en mathématiques, statistique, biologie, informatique, sciences de l'ingénieur \\
& 9 en économie, comptabilité, gestion, droit \\
\hline Corps d'Etat & 6 certifiés \\
& 5 agrégés \\
& 14 maîtres de conférences \\
& 1 professeur des universités \\
\hline Sexe & 13 hommes \\
& 13 femmes \\
\hline Cursus & 2 parcours CPGE + ENS (+ doctorat pour un enquêté) \\
& 16 parcours universitaires purs dont 2 DUT en début \\
& 1 parcours BTS + diplômes professionnels \\
& 5 parcours CPGE + Ecole d'ingénieur + Doctorat pour 4 enquêtés \\
& 1 parcours CPGE + université (maitrise) \\
& 1 parcours DEUG + Ecole d'ingénieur + maitrise + doctorat \\
\hline PCS du père & 2 Agriculteurs exploitants \\
& 8 Artisans, Commerçants et Chefs d'entreprise \\
& 10 Cadres et Professions Intellectuelles Supérieures \\
& 3 Professions Intermédiaires \\
\hline Effectif total & 3 Ouvriers \\
\hline Ancienneté à I'IUT en 2013 & 5 inférieure ou égale à 3 ans \\
& 10 entre 4 et 10 ans \\
& 11 entre 11 et 20 ans \\
\hline & \\
\hline
\end{tabular}

Sigles : BTS (Brevet de technicien supérieur) ; (PGE (Classe préparatoire aux grandes écoles) ; DEUG (Diplôme d'études universitaires générales) ; DUT (diplôme universitaire de technologie) ; ENS (Ecole normale supérieure) ; PCS (Professions et Catégories socioprofessionnelles selon l'INSEE, source https://www.insee.fr/).

Source : Enquête ethnographique au sein de l'IUT étudié, année 2012 - 2013.

\section{Le potentiel socialisateur des IUT}

Si l'histoire des IUT reste à ce jour largement à écrire, on peut préciser rapidement que leur création, au milieu des années soixante, s'appuie sur une "doctrine de l'enseignement supérieur technique " (Bernard, 1970, p. 15 ; Dormoy-Rajraman, 2015). Cette doctrine se matérialise, entre autres, par une série d'injonctions à organiser des relations entre l'enseignement supérieur (via les IUT) et le monde économique (Chambart et Le Cozanet, 2015). Il s'agit ici de présenter, dans un premier temps, la vision et l'organisation de ces relations sur le terrain étudié. Dans un deuxième temps, nous évoquons les éléments théoriques permettant d'analyser la socialisation à l'œuvre. Dans un troisième temps, l'attention est portée sur les éléments du cadrage institutionnel du tutorat en s'intéressant aux devoirs du tuteurs. 


\subsection{Un IUT « hyperprofessionnaliste »}

Dans le cas de l'IUT étudié, la socialisation aux activités de professionnalisation des étudiants constitue un ensemble justifié par un "projet d'établissement " fort, entendu comme une "colonne vertébrale ", un élément "structurant ", ${ }^{7}$ qu'il s'agit de présenter rapidement.

Créé en 1992 et situé à proximité d'une grande ville de province, cet IUT s'inscrit dans l'objectif de revalorisation de l'apprentissage, et y souscrit largement, suite aux lois de 1987 et 1992 (Moreau, 2003, chapitre 1 ; Kergoat et Capdevielle-Mougnibas, 2013), en s'appuyant sur un contexte de normalisation de "la " relation "formation-emploi " (Tanguy, 2002), de dégradation des conditions d'entrée dans la vie active des jeunes et des conditions de travail, d'arrivée dans l'enseignement supérieur de cohortes issues de la seconde explosion scolaire.

L'institut va alors décliner, dans son organisation, une vision de l'apprentissage comme outil de démocratisation des études supérieures : il doit permettre à des jeunes qui n'auraient pu l'obtenir autrement, l'accès à un diplôme de l'enseignement supérieur et à l'emploi. Le schème de perception de "l'entreprise formatrice " (Moreau, 2003, p. 36) est très présent sur ce terrain. D’une manière générale, l'entreprise, le privé, sont parés de valeurs positives qu'étudiants comme futurs enseignants doivent savoir énoncer au moment de leur propre recrutement (Tralongo, 2015a).

L'ensemble s'organise autour d'une pédagogie de l'alternance qui a été maintenue par les trois équipes de direction successives. L'alternance (majoritairement par contrat d'apprentissage) est alors la seule modalité de formation proposée aux six-cents alternants, inscrits en 2012-2013, dans l'un des cinq DUT ou les sept licences professionnelles ${ }^{8}$ que compte cet IUT. On peut ainsi qualifier d'intensive la relation des étudiants à l'entreprise au cours de leur formation dans cet IUT.

Pour l'IUT étudié, la finalité est ainsi d'amener des étudiants (plutôt scolairement très moyens) à être "employables » dès l'issue du DUT, ou après une année d'études supplémentaire, dans un domaine professionnel en lien avec les diplômes obtenus. Et pour cela, titulaires, vacataires, personnels administratifs, d'enseignement et de recherche, ont un rôle à jouer que l'IUT va s'efforcer d'encadrer.

7. Les trois expressions relèvent du vocabulaire indigène.

8. Les domaines professionnels des diplômes préparés au sein de cet IUT concernent : la gestion des entreprises et des administrations (DUT GEA) ; le transport et la logistique (DUT GLT) ; la qualité, logistique industrielle et l'organisation (DUT QLIO) ; la statistique et l'informatique décisionnelle (DUT STID) ; la sécurité, l'hygiène et l'environnement (DUT HSE). Les licences professionnelles sont rattachées à ces différents domaines. 


\section{2 L'activité tutorale : entre injonctions institutionnelles et appropriations}

L'objet de cette recherche est de saisir "comment les individus sont socialisés concrètement dans le cadre de l'exercice professionnel lui-même, tout au long de la carrière " (Zolesio, 2012, p. 378). Cette socialisation est ainsi envisagée comme un "processus dynamique " réalisé au cours de l'activité professionnelle (Alain \& Pruvost, 2011, p. 269). Résultant de confrontations, frottements et ajustements entre institutions et individus, la socialisation est "la façon dont la société forme et transforme les individus ".?

L'ensemble s'appuie sur une approche "dispositionnaliste-contextualiste " (Lahire, 2012, pp. 21-22). Les acteurs sont vus comme dotés de propriétés sociales incorporées. Lorsqu' ils commencent à travailler en IUT, tout un ensemble d'institutions (la famille, l'école, les institutions de formation et de socialisation professionnelle, les pairs, ...) a déjà produit certaines dispositions (constituées de goûts, cultures, pratiques, savoirs, rapport au pouvoir, au langage, au travail, etc.) rendant possible le fait qu'ils soient tout d'abord autorisés à être recrutés dans ce type d'institution, puis qu'ils y travaillent en mobilisant certaines manières de faire plutôt que d'autres. Il s'agit à la fois d'en rendre compte, et de questionner ce qui a pu être mobilisé en tant que ressource ou non pour devenir diversement partie prenante de la professionnalisation des étudiants en IUT.

Par ailleurs, l'IUT au sein duquel les acteurs vont travailler est à envisager aussi dans une perspective socio-historique (Noiriel, 2008), dans son emboîtement avec d'autres institutions (les IUT en France, l'Université, l'enseignement supérieur français et européen), dans son organisation (Musselin, 2008). Il développe des dispositifs, des instruments, des outils, des valeurs, des normes qui sont à mettre au jour, l'ensemble étant susceptible d'avoir une force de cadrage sur les acteurs.

Des travaux en sociologie du management (Courpasson, 2000), de l'administration publique (Lascoumes et Le Galès, 2005 ; Buisson-Fenet \& Le Naour, 2008), de l'éducation (Bonnéry, 2014) soulignent en effet l'intérêt d'étudier finement les outils, dispositifs et matériels pédagogiques, considérés comme relayant et matérialisant des injonctions pouvant occasionner des appropriations diversifiées par des acteurs.

Pour rendre compte de ces appropriations, il faut s'intéresser au travail de confrontation mené dans le contexte sur et par l'acteur. L'approche ethnographique a permis de suivre des titulaires à différents moments de leur carrière et à s'intéresser aux façons dont ils apprennent les gestes, techniques, etc., attendus lors des activités dites de "professionnalisation" des étudiants.

9. Darmon, 2006, p. 6. 
La notion d'appropriation (de Certeau, 1990 ; Chartier, 1992), en postulant une variation entre les injonctions et ce qu'en feront des individus socialement situés, permet d'analyser les pratiques comme des résultats de confrontations : "La notion d'appropriation permet ainsi d'appréhender à la fois la diversité des pratiques et des usages sociaux et la complexité de situations sociales dans lesquelles les individus ou les groupes sociaux ne sont pas seulement condamnés à se soumettre aux normes ou aux logiques qui président à ces situations mais peuvent se saisir de celles-ci à partir de leurs propres logiques. " (Thin, 2010, p. 75)

\subsection{Du côté du cadrage : les devoirs du tuteur}

D’un point de vue juridique, tout apprenti doit être encadré par un maître d'apprentissage dont les missions sont définies par la loi (Article L6223-5 du Code du travail). Celui-ci est le référent direct de l'apprenti dans l'entreprise. Le Centre de formation d'apprentis (CFA) dont dépendent les Unités de formation par apprentissage regroupées dans l'IUT étudié, prévoit un référent également du côté de la formation, aux appellations diverses, telles que "tuteur pédagogique ", "tuteur UFA ", etc. (Dubrion, 2006). Il s'agit, dans cet IUT, du « tuteur IUT».

Ce que doit faire le «tuteur IUT» relève tout d'abord de rencontres entre stagiaire/alternant, maître de stage/d'apprentissage, tuteur IUT. Ces rencontres doivent se dérouler en entreprise, en présence des trois protagonistes ; comporter une partie de présentation par l'alternant de son travail, une partie d'évaluation de son travail ; se tenir à des périodes fixées par l'IUT ; aboutir au remplissage d'une grille évaluation dans le cadre produit par l'IUT, avec des items à remplir et barèmes de notations. Ainsi, le tuteur a pour mission de se déplacer pour la période d'apprentissage (qui dure une année), cinq fois en entreprise pour le DUT, quatre fois pour la licence professionnelle.

Ensuite, le travail concerne l'encadrement du mémoire de l'alternant, mais ce point, qui renvoie à des activités plus traditionnelles d'un enseignant-chercheur ${ }^{10}$, n'est pas traité dans l'article. Enfin, le tuteur doit participer, à ce titre, aux jurys de diplôme.

Le «tuteur IUT» prend connaissance de ses devoirs via différentes sources : les membres des "partenariats entreprise " ${ }^{11}$, en affectant tel tuteur à tel alternant, jouent un rôle pour transmettre les attendus institutionnels. La transmission passe par un ensemble de formations collectives (généralement une journée à l'IUT, regroupant également

10. Il s'agit pour le tuteur d'aider l'alternant dans la réalisation de son mémoire (aide à la définition de la problématique, des hypothèses, de la bibliographie, de l'élaboration d'un plan, ...).

11. Implantés au sein de chaque département, les services «Partenariat-entreprise " comptabilisent un ou plusieurs "membres " (administratifs ou enseignants, ayant généralement exercé une activité salariée de longue durée dans le privé), dont l'une des missions concerne l'affectation, la formation des tuteurs ainsi que le suivi du travail de tutorat. 
des maîtres d'apprentissage, conjointement organisée par le CFA et l'IUT), individuelles (en face-à-face avec un membre du "partenariat-entreprise »). Un voir-faire est également proposé : concrètement, le novice accompagne un tuteur expérimenté lors de visites en entreprises en tant qu'observateur, puis il réalise lui-même en solo des tutorats. Un apprentissage par le faire parachève la prise en main de la mission tutorale.

Des instruments et outils sont également mobilisés. Un document papier, le « Livret de suivi de l'alternant", se compose d'une soixantaine de pages mêlant informations, indications et rubriques à remplir. À la date de l'enquête, ce livret était commun aux DUT et spécifique à chaque licence professionnelle pour une partie de son contenu. Il s'agit d'un document produit pour différents récepteurs et distribué à l'alternant, le tuteur et le maître d'apprentissage. L'intention institutionnelle est que toutes les parties prenantes aient le même discours, mêlant des éléments de présentation et d'information sur l'IUT, sa "philosophie de l'alternance ", portant sur les "rôles " des tuteurs, les moments et les lieux d'évaluation. Il est ainsi stipulé que "le rôle du tuteur est d'animer les différentes rencontres en entreprise, et d'apporter un avis sur la qualité des situations de travail proposées dans une perspective de formation de l'alternant et sur leur cohérence avec les objectifs du diplôme. " ${ }^{12}$

Les discours sur la professionnalisation, les livrets et les formations correspondent à un ensemble de dispositifs, instruments et outils (Bonnéry, 2014 ; Tralongo, 2015b) visant la fabrication d'un cadre homogénéisant la perception et les pratiques tutorales. Cette fonction semble converger avec celle mise en évidence par le "Portefeuille d'Expérience et de Compétences" (Beaupère \& ali., 2014). Reste à voir ce que cela produit chez les enquêtés.

\section{Devenir tuteur : entre l'expert et le 2 déclencheur d'échanges}

L'activité tutorale concerne de fait tous les enquêtés. Ils semblent en cela s'approprier de façon orthodoxe une injonction officielle de participation aux activités de professionnalisation des étudiants. Dans cet Institut, une spécificité consiste à ne pas cloisonner les interventions tutorales par diplôme : concrètement, les titulaires sont invités à encadrer des alternants dans les différents diplômes proposés par cet Institut, qu'ils y enseignent ou non. Dès lors, pour la suite, nous nous intéressons aux pratiques tutorales effectuées au sein des douze diplômes que compte cet IUT.

Une partie des entretiens était consacrée à faire relater la façon dont a débuté cette activité, les choix réalisés au cours de la carrière (concernant les types de diplômes, les

12. IUT, Livret de suivi et d'évaluation, année 2012-2013, p. 6. 
entreprises, etc.), les anecdotes frappantes, ainsi que les perceptions pour cette activité, les projections pour la suite.

Chaque enquêté évoque entre deux et huit suivis d'étudiants par an, ce qui signifie des déplacements en entreprise, de dix à quarante fois par an. Pour tous les enquêtés, on compte au moins deux diplômes d'intervention sur une même année, et quatre à six au cours de leur carrière (combinant des DUT et des licences professionnelles) sur les douze proposés dans cet Institut.

Il est à noter que cette activité est comptabilisée dans leur « service » d'enseignement ${ }^{13}$. Cette précision est importante car elle indique que l'activité tutorale est visible, localement légitimée, et ne consiste pas en un surcroît de travail au regard de l'enseignement, contrairement à ce qui a pu s'observer en d'autres lieux (Losego, 2004). Cette activité n'est néanmoins pas assimilée à de l'enseignement, car aucun enquêté n'emploie ce terme pour la qualifier. Cela montre une première appropriation de la vision institutionnelle du tutorat et justifie la question sur la familiarisation à une activité positionnée comme en décalage par rapport à l'enseignement et la recherche.

Les réponses permettent de différencier les manières d'avoir commencé le tutorat, que nous avons scindées d'abord en deux groupes : les débuts déclarés "faciles » ou "impressionnants ", qui sont analysés en termes de proximité et distance, autrement dit de plus ou moins grande distance d'expérience sociale entre les univers académique et économique. Dans un second temps, nous distinguons deux rôles joués par les tuteurs : le déclencheur d'échanges ou l'expert.

13. La prise en compte dans le service est la suivante : un suivi d'étudiant en stage correspond à cinq heures d'enseignement en travaux dirigés, tandis qu'un suivi d'un alternant sur une année en apprentissage correspond à douze heures. Les volumes horaires importants nécessaires à cette politique étaient financés par l'alternance. 
Tableau 2 : Répartition des enquêtés selon les discours sur les débuts dans le tutorat

\begin{tabular}{|c|c|}
\hline & Tutorat \\
\hline $\begin{array}{l}\text { Débuts « faciles », « évidents", } \\
\text { " naturels » }\end{array}$ & $\begin{array}{l}\text { Groupe } A \text { - } 20 \text { enquêtés } \\
\text { Disciplines : Sciences de gestion, sciences économiques, informatique/sciences de l'ingénieur ; } \\
\text { sciences de l'éducation ; statistiques ; langues } \\
\text { Corps d'Etat : } 6 \text { certifiés ; } 4 \text { agrégés ; } 10 \text { MCF ; } 1 \text { PU } \\
\text { Sexe : } 12 \text { H ; } 8 \text { F } \\
\text { Parcours de formation : } \\
12 \text { parcours universitaires « purs » } \\
5 \text { parcours Ecole d'ingénieur + Doctorat pour } 4 \text { enquêtés } \\
1 \text { parcours BTS + diplômes professionnels } \\
1 \text { parcours CPGE + université (maîtrise) } \\
1 \text { parcours DEUG + Ecole d'ingénieur + maîtrise + doctorat } \\
\text { PCS: } \\
2 \text { Agriculteurs exploitants } \\
8 \text { Artisans, Commerçants et Chefs d'entreprise } \\
8 \text { Cadres et Professions Intellectuelles Supérieures } \\
2 \text { Ouvriers }\end{array}$ \\
\hline $\begin{array}{l}\text { Débuts « pas évidents » et/ } \\
\text { ou qui « interpellent», } \\
\text { « questionnent» }\end{array}$ & $\begin{array}{l}\text { Groupe B - } 6 \text { enquêtés } \\
\text { Disciplines : Biologie, sociologie, statistique ; mathématique ; expression communication } \\
\text { Corps d'Etat : } 4 \text { MCF ; } 2 \text { PRAG } \\
\text { Sexe : } 1 \text { H ; } 5 \text { F } \\
\text { Parcours de formation : } \\
2 \text { parcours CPGE + ENS (+ doctorat pour un enquêté) } \\
4 \text { parcours universitaires « purs » } \\
\text { PCS: } 2 \text { Cadres et Professions Intellectuelles Supérieures } \\
3 \text { Professions Intermédiaires } \\
1 \text { Ouvriers }\end{array}$ \\
\hline
\end{tabular}

Sigles : BTS (Brevet de technicien supérieur) ; (PGE (Classe Préparatoire aux Grandes Ecoles) ; DEUG (Diplôme d'études universitaires générales) ; DUT (Diplôme universitaire de technologie) ; ENS (Ecole Normale Supérieure) ; MCF (Maître de Ccnférences) ; PCS (Professions et Catégories socioprofessionnelles, selon I'INSEE, source https://www.insee.fr/) ; PRAG (Professeur Agrégé) ; PU (Professeur des Universités).

Source : Enquête ethnographique au sein de I'IUT étudié, année 2012 - 2013.

\subsection{Des débuts « faciles »}

Les débuts dans le tutorat sont relatés comme fluides pour les vingt enquêtés du groupe A. Les propos évoquent plusieurs raisons, qu'on envisage comme autant d'éléments ayant joué en tant que ressources dans cette situation. Ces ressources concernent les expériences professionnelles antérieures des enquêtés (dans le privé, dans des lycées en tant qu'enseignant en Section de techniciens supérieurs) ainsi qu'une socialisation au monde professionnel privé par le biais de la parentèle.

Avant leur recrutement à l'IUT, il est tout d'abord à noter que ces enquêtés ont une expérience du travail dans le privé par l'occupation de postes, soit en tant qu'étudiant sous des statuts plutôt protégés (stagiaire d'école d'ingénieurs, de DESS ${ }^{14} /$ Master professionnel), soit en tant que salarié de longue durée dans le privé. La familiarisation au monde du privé

14. Diplôme d'études supérieures spécialisées. 
a donc déjà eu lieu et elle fournit aux titulaires un ensemble de ressources mobilisables dans le contexte du tutorat. Celles-ci sont constituées de dispositions, de savoirs sur les contenus de postes et de missions, de savoir-faire pour discuter (négocier) avec leurs interlocuteurs. En mobilisant des connaissances techniques, épistémiques, le tuteur dialogue avec les alternants et maittres d'apprentissage sur le contenu opérationnel des missions, encadre l'alternant sur cet aspect de son travail. Ces enquêtés se positionnent en experts, autrement dit en pourvoyeurs de solutions pour les entreprises, qu'il s'agit de satisfaire.

Ainsi un enquêté explique :

"Bon déjà, le monde de l'entreprise, je connaissais, donc je n'avais pas de peur particulière à ce sujet. L'essentiel de ma tâche de tuteur, c'est de s'assurer que l'étudiant arrive à amener, d'un point de vue opérationnel, un résultat qui atteint la satisfaction de l'entreprise. Et qui tienne compte des objectifs de la formation."

Pour cela, cet enquêté préfère choisir d'encadrer des missions pour lesquelles il peut avoir "un certain recul technique.» (enseignant-chercheur en informatique, 8 ans d'ancienneté, formation en école d'ingénieur, père cadre dans le privé)

Une autre expérience concerne les enquêtés de ce groupe, qui sont issus du second degré : ceux qui ont enseigné en BTS avant d'être en poste dans cet IUT relatent avoir déjà eu ce type d'activité et de relations aux entreprises lorsqu'ils étaient en lycée. Ainsi, cet enquêté qui explique :

"Quand jétais en BTS, il y avait des stages. Il y avait un rapport de stage à faire à la fin. Il y avait des visites, mais cétait moins cadré quici. " (enseignant certifié en comptabilité-gestion, 6 ans d'ancienneté, père ouvrier)

Un autre type d'expérience est à évoquer, qui n'est pas exclusive des précédentes : il s'agit d'une socialisation au privé par le biais du travail de la parentèle des enquêtés (huit enquêtés). Ces enquêtés relatent, entre autres, l'acquisition d'une sensibilité pour la "relation client " apprise au sein de l'entreprise familiale (un garage, une épicerie-restaurant, différents petits commerces, une entreprise de transport). De plus, certaines des activités, étudiées et pratiquées par leurs étudiants, telles que la comptabilité, la gestion, etc., sont connues par les enquêtés car ils les ont eux-mêmes réalisées ou ont vu leurs parents, leur conjoint, les mener. Elles sont donc loin d'être uniquement scolaires ou académiques pour eux.

D'une façon synthétique, les enquêtés de ce groupe considèrent que cette activité de tutorat est "essentielle » et " complémentaire " non seulement à l'enseignement, mais également à leur activité de recherche. Cela leur permet de voir " où on en est dans l'entreprise " par rapport à telle ou telle notion théorique, problématique, méthodologie, ou encore technique. Pour cette enquêtée, le tutorat est "énormément apprécié. Je me suis tout de suite sentie à l'aise. C'est par ce biais que je sonde le mieux ce qui se passe dans l'environnement professionnel. " (enseignante-chercheuse, sciences économiques, parcours universi- 
taire, plusieurs contrats à durée indéterminée dans le privé, 13 ans d'ancienneté, parents artisans-commerçants)

On retrouve dans ce groupe toutes les disciplines pour lesquelles les entreprises, les missions des alternants peuvent constituer des "terrains" de recherche, d'élaboration de cas pratiques et d'exemples ou appuis pour les cours : sciences de gestion ; sciences de l'ingénieur ; didactique professionnelle, productique, informatique, statistique, langues.

Néanmoins, il faut souligner que connaître le monde de l'entreprise ne suffit pas pour savoir comment être un tuteur dans le sens conféré par cet IUT. Sur ce point, les enquêtés des deux groupes relatent un apprentissage réalisé à partir de différents dispositifs, instruments et outils institutionnels, dont le « livret» (développé plus bas).

\subsection{Un apprentissage progressif multidimensionnel}

Les démarrages pour le groupe B (six enquêtés) relèvent, à l'inverse, d'un ensemble de sentiments de décalages et d'étrangetés au niveau : des codes de conduites (relationnel, vestimentaire) à avoir en entreprise ; du rôle à tenir, de la posture attendue ; des technologies et savoirs professionnels dans l'entreprise ; du discours de présentation de l'IUT.

Ce ne sont pas des "difficultés insurmontables", ainsi que le déclare une enquêtée, mais des questions qui se posent, des gênes qui peuvent conduire à des souvenirs suffisamment forts pour être rapportés précisément lors de l'entretien. L'acculturation consiste en un saut à réaliser, plus grand que dans le cas des enquêtés du groupe $\mathrm{A}$.

Ceux qui relatent une certaine étrangeté dans les débuts du tutorat ont une ou plusieurs des caractéristiques suivantes : leur formation est purement universitaire (pas d'incursion du côté des grandes écoles) ; ils n'ont eu que marginalement l'occasion d'entrer dans l'entreprise au cours de leur formation ; lorsqu'ils sont enseignants-chercheurs, leurs sujets de thèse n'était pas en lien avec des thématiques de l'entreprise; ils ont une parentèle majoritairement issue de la fonction publique ; les «jobs " alimentaires et étudiants ont été réalisés dans des structures publiques.

Ce qui se matérialise ici, c'est une certaine distance en termes d'expériences, de dispositions et de familiarité avec le monde du privé, avec les technologies mobilisées en entreprise, et le contenu des activités demandées aux alternants.

Ainsi, une enquêtée raconte :

"Au premier rendez-vous, jétais impressionnée, j’avais du mal à trouver ma place en entreprise. J'avais un déficit, j'avais pas l'impression d'être dans mon élément. J'avais l'impression d'être dans un film américain, on m'attendait à l'accueil, il avait un grand ascenseur...". (enseignante-chercheuse, sciences sociales, quelques mois d'ancienneté au moment de l'entretien, parcours universitaire, parents de profession intermédiaire dans le public). Elle relate avoir réfléchi à sa manière de s'habiller : "oui, je me suis posé beaucoup de questions, 
je ne voulais pas en faire trop ". Après quelques mois de tutorat, elle dit qu'elle continue à se poser des questions, sur le rôle et la fonction du tuteur :

" Je viens pour valider, mais qu'est-ce que j’apporte? Je n'ai pas l'impression de servir à grand-chose."

Pour ces enquêtés, leur manière de démarrer dans la fonction tutorale consiste à jouer le rôle (attendu institutionnellement) d'un déclencheur d'échanges : lorsqu'ils se rendent en entreprise pour l'évaluation de l'alternant, ils coordonnent, animent des discussions et évaluations entre le maître d'apprentissage et l'alternant, sans toutefois mobiliser le rôle d'expert précédemment évoqué. Il est intéressant de constater qu'au fil des ans, un basculement dans le rôle d'expert peut être relaté par ces enquêtés, qui vont se "spécialiser » dans certains domaines de métiers.

Cette spécialisation est observée lorsque les enquêtés prennent des responsabilités administratives dans certains diplômes. La socialisation aux fonctions tutorales devient alors aussi une familiarisation à certaines problématiques, connaissances et technologies en vigueur dans les domaines professionnels visés par les diplômes.

À l'inverse, on constate également que lorsque les enquêtés du groupe A font du tutorat pour des diplômes éloignés de leur domaine disciplinaire de compétence, ils déclarent ne pas pouvoir mobiliser un rôle d'expert et en rester à un rôle de déclencheur d'échanges. Pour certains, ce rôle est considéré comme inconfortable et non satisfaisant. Ainsi, une enquêtée (dira à propos d'un tutorat dans le milieu bancaire :

"Intérieurement, j'étais frustrée, je n'apportais pas grand-chose aux entreprises, j’étais juste la tutrice qui vient évaluer. » (enseignante-chercheuse, informatique, treize ans d'ancienneté, formation d'ingénieur, père industriel)

\subsection{Un tuteur tutoré}

Le "livret de suivi de l'apprenti » va être cité par tous les enquêtés (groupe A et B) comme un point d'appui solide et incontournable. Celui-ci va être "parcouru ", "lu », ou "étudié» diversement, mais il est toujours évoqué comme ayant joué un rôle structurant.

À côté du livret, un étayage a été également souvent organisé et les enquêtés relatent comment ils ont commencé par accompagner des tuteurs plus expérimentés, avant de se "lancer» dans leur premier rendez-vous en entreprise. L'étayage a généralement été produit par des membres des "partenariats entreprises ». La situation vécue par une enquêtée, qui appartient au groupe des débuts difficiles, est ainsi assez typiquement relatée: "Avant mon premier tutorat, je suivais $P$. [un $P A S T^{15}$ ], qui était tuteur. Il mavait impressionnée, il était comme un poisson dans l'eau, je l'ai suivi à chaque fois. Je le regardais faire, jécoutais et après je pouvais reproduire seule en entreprise. Je l'ai suivi sur les cinq rendez-vous. " (ense-

15. Professeur associé à statut temporaire. 
gnante-chercheuse en informatique, 16 ans d'ancienneté à l'IUT, parcours universitaire, parents enseignants)

À cet étayage s'ajoutent des formations informelles (en face-à-face), des " discussions " avec des membres des services "Partenariat-Entreprise», et plus rarement des formations formelles.

Enfin, soulignons le rôle de certains maîtres d'apprentissages, qui apprennent au tuteur à l'être. Ainsi cette enquêtée qui déclare :

"Elle [un membre du service Partenariat Entreprise] m'avait donné du tutorat. Alors j'avais dit, le tutorat, c'est quoi ? Et elle m'avait dit "tinquiète pas, je vais te donner du tutorat chez $X$., dans le domaine du transport, ce sont des habitués. Ils ont des apprentis chaque année, les missions sont bien balisées, elles ne changent pas trop, les maîtres d'apprentissage aussi ont l'habitude, ce n'est pas la première fois qu'ils font du tutorat". Et puis j'ai été bien guidée malgré tout par le petit livret. Et après, j'ai fait du tutorat hors de cette entreprise. " (enseignantechercheuse en sciences économiques, formation universitaire, 8 ans d'ancienneté, parents commerçants)

Ainsi, pour saisir le travail d'appropriation, nous avons utilisé comme indice de positionnement de départ un couple d'opposition entre proximité et distance envers le monde économique dans les situations vécues. On peut, d'une part, noter que ces qualificatifs de familiarisation ne sont pas mécaniquement déductibles d'une seule variable (le type d'études, la discipline, l'origine sociale, etc.). L'analyse doit en effet passer par la reconstruction de configurations individuelles et l'observation de données fines (par exemple, la discipline étudiée ne suffit pas, il faut aussi s'intéresser au sujet de thèse, au terrain, aux lieux de formation, etc.). Soulignons, d'autre part, que la distance n'est pas une donnée figée : les entretiens relatent des apprentissages réalisés au cours des pratiques professionnelles, dans un cadre ici institutionnellement organisé pour qu'ils aient lieu. Au cours de la pratique, l'activité tutorale est appropriée au moyen de différents outils et instruments, dans des contextes variés (à l'IUT, dans des entreprises) et au contact d'acteurs extérieurs au monde académique (des membres de service "partenariat entreprise ", des maîtres d'apprentissage, des PAST).

Le tutorat est alors perçu comme "intéressant", car il permet " de se rendre compte de la diversité des missions, des situations de travail» des apprenants. Au cours des années, l'activité « plaît» et elle est conservée. Son évocation mobilise le champ lexical de l'intérêt, des émotions agréables et positives, et cette remarque vaut pour les enquêtés des deux groupes. Ainsi, peu à peu, leurs propos se rejoignent et, surtout, rejoignent le discours officiel : les ajustements du départ ont été réalisés, et en produisant des discours plutôt convergents, ils emblent attester d'un cadrage institutionnel assez fort. 


\section{Conclusion}

Si la professionnalisation a pu être considérée comme une des injonctions marquantes dans le déploiement du processus de Bologne (Agulhon \& ali., 2012), ses modalités de mise en œuvre sur des terrains diversifiées, tout autant que ses effets sur les acteurs de l'enseignement supérieur restent largement à étudier. L'ambition de cet article était de poser la question des effets socialisateurs de la professionnalisation sur les enseignants et enseignants-chercheurs. L'entrée choisie était l'activité tutorale qui consiste, au sein d'un IUT étudié de façon monographique, en un suivi d'alternants en entreprise par des enseignants.

Lanalyse permet de montrer que cette socialisation est cadrée par tout un appareillage (discours, dispositifs, instruments, outils, étayages) visant à articuler un discours professionnaliste avec des pratiques pédagogiques organisées à partir de situations d'alternance. Cet ensemble semble tirer son efficacité (du point de vue indigène) en agissant sur et avec des titulaires qui possèdent déjà certaines dispositions, des savoirs et connaissances sur les métiers pour lesquels se forment les alternants.

Deux groupes d'enquêtés ont été repérés à partir des récits de débuts dans le tutorat (signifiant sur ce terrain un suivi d'alternant en entreprise). Que le groupe des enquêtés évoquant des débuts " faciles» dans le tutorat soit plus nombreux que celui évoquant des démarrages "impressionnants" n'est pas un hasard et renvoie à des stratégies précises de recrutement (Tralongo, 2015b) : l'enjeu pour cet IUT n'est pas d'avoir à produire, chez les titulaires une socialisation de conversion (Darmon, 2006), mais plutôt des rééquilibrages, des ajustements, fondés sur un existant plutôt convergent avec les attentes institutionnelles.

De sorte que la double coupure énoncée comme hypothèse en introduction (une absence de familiarisation aux formations en IUT et de liens entre les métiers de l'enseignement et de la recherche et ceux préparés par les étudiants) est à nuancer. Si effectivement les diplômes et leur fonctionnement en IUT sont découverts lors de l'insertion dans la vie professionnelle des enquêtés (et non en tant qu'étudiants), bien des éléments dans leur propre parcours de formation et de premières expériences professionnelles relativisent l'idée d'une expérience tutorale comme radicale étrangeté. Les formations en école d'ingénieurs, l'inscription des parcours professionnels des enquêtés et de la parentèle dans le privé, dans l'artisanat et le commerce, les proximités dans les cultures, les technologies disciplinaires tendent à produire les ingrédients d'une familiarisation préalable. Lorsque cette familiarisation est construite pendant les premières années de travail au sein de cet IUT, on constate que les discours se font écho pour redoubler le discours institutionnel et produire une perception positive concernant cette activité. Les enquêtés semblent ainsi plutôt bien ajustés.

Pour conclure, soulignons que la distance initiale forte de certains enquêtés avec le privé n'est pas synonyme de vide, car elle signifie des expériences dans des univers moins directement reliés au monde économique. C'est la confrontation à une situation particulière, qui 
fait surgir un sentiment de familiarité ou d'étrangeté et qui en outillant certains enquêtés à aller «facilement » en entreprise, dote ainsi certaines expériences antérieures d'une valeur positive et les transforme en ressources. Le risque serait d'en rester à un regard privatif, qui rend invisible et nul ce qui n'apparaît pas comme mobilisable dans le contexte attendu. Et de ne pas voir combien cette socialisation est un travail, coûteux, tant institutionnellement, individuellement, que financièrement (ici les dispositifs et postes d'administratifs sont largement financés via les ressources de l'alternance), y compris lorsque toutes les conditions semblent favorables.

Il y a pourtant des enjeux à garder cette remarque à l'esprit. Les récents prolongements de la loi sur l'enseignement supérieur (2013) mettent en avant une volonté de développer les stages à l'université et de renforcer leur "dimension pédagogique» : "La loi prévoit que le stage soit pensé en amont et évalué en aval en fonction d'objectifs pédagogiques clairement définis. Il doit également bénéficier obligatoirement d'un double suivi, par un enseignant et par un tuteur au sein de l'organisme d'accueil, afin d'accompagner l'acquisition de véritables compétences. ${ }^{16}$ " Dans cette perspective, et au regard de l'énergie et du coût déployés par l'IUT étudié pour faire vivre un dispositif de rapprochement entre les mondes académique et économique, cela invite à se poser la question des conditions institutionnelles (aussi bien matérielles, financières, organisationnelles, identitaires, ...) ainsi que des effets sur les acteurs d'un tel rapprochement, dans des composantes universitaires dotées d'autres cultures, histoires et pratiques d'enseignement et de recherche (Faure \& al., 2006).

\section{Bibliographie}

ADIUT (2007), Livre blanc sur le système IUT : après 40 ans d'existence : histoire, bilan, perspectives, Assodiut, [en ligne], $60 \mathrm{p}$.

Agulhon C., Convert B., Gugenhiem F., Jakubowski S. (2012), La professionnalisation. Pour une université " utile "?, Paris, L'Harmattan.

Alain M., Pruvost G. (2011) " Police : une socialisation professionnelle par étapes ", Déviance et Société, Vol. 35.

Alam T., Gurruchaga M., O’Miel J. (2012), «Science de la science de l'état : la perturbation du chercheur embarqué comme impensé épistémologique ", Sociétés Contemporaines, $n^{\circ} 87$.

Allouch A., Van Zanten A. (2008), "Formateurs ou "grands frères" ? Les tuteurs des programmes d'ouverture sociale des Grandes Écoles et des classes préparatoires », Education et Sociétés, $\mathrm{n}^{\circ} 21$.

16. http://www.gouvernement.fr/action/l-encadrement-des-stages. 
Beaupère N., Bosse N., Lemistre P. (2014), « Expérimenter pour généraliser le Portefeuille d'Expériences et de Compétences à l'université : le sens de l'évaluation », Formation Emploi, $\mathrm{n}^{\circ} 126$.

Bernard M.-Y. (1970), Les Instituts Universitaires de Technologie, Paris, Dunod.

Bonnery S. (2014), De l'étude des inégalités scolaires à celle de la domination scolaire. Pertinence de la dimension méso-sociologique en sociologie de l'école, Mémoire d'Habilitation à Diriger des Recherches, Paris 8.

Bourdon J., Guégnard C., Michot C. (2014), « La sécurisation des parcours des apprentis à l'épreuve du choix politique ", Formation Emploi, n 126.

Buisson-Fenet H. \& Le Naour G. (2008), Les professionnels de l'action publique face à leurs instruments, Toulouse, Octarès.

Certeau de M. (1990), L’invention du quotidien. T1. Arts de faire, n 146, Paris, Gallimard.

Chambard O., Cozanet (Le) L. (2015), "Introduction : Nouveaux éclairages sur les relations entre enseignement supérieur et monde économique ", Formation Emploi, n 132.

Chartier R. (1992), L'ordre des livres. Lecteurs, auteurs, bibliothèques en Europe entre XIVe et XVIIIe siècle, Aix-en-Provence, Alinéa.

Courpasson D. (2000), L'action contrainte. Organisations libérales et domination, Paris, PUF.

Darmon M. (2006), La socialisation, Paris, La Découverte.

Demazière D. (2009), " Postface. Professionnalisations problématiques et problématiques de la professionnalisation ", Formation Emploi, n ${ }^{\circ} 108$.

Dormoy-Rajramanan C. (2015), "L'ouverture de l'Université vers le monde extérieur autour de 1968 : entre consensus partiel, polarisation et compromis ", Formation Emploi, $\mathrm{n}^{\circ} 132$.

Dubar C. (1991), La socialisation, construction des identités sociales et professionnelles, Paris, Armand Colin.

Dubrion B. (2006) "Qu'est-ce qu’un "bon apprenti" du supérieur? ", Formation Emploi, $\mathrm{n}^{\circ} 96$.

Faure S., Soulié C. (2006), "La recherche universitaire à l'épreuve de la massification scolaire ", ARSS, 4, n 164 .

Hugues E. C. (1996), Le regard sociologique, EHESS, Paris.

Kergoat P., Capdevielle-Mougnibas V. (2013). "Les formations par apprentissage : un domaine de recherche à développer ", Revue Française de Pédagogie, 183, pp. 41-42. 
Lahire B. (2012), Monde pluriel, Penser l'unité des sciences sociales, Paris, Seuil, coll. "La couleur des idées ".

Lascoumes P., Le Galès P. (2005), Gouverner par les instruments, Paris, Presses de Sciences Po.

Losego P. (2004), "Le travail invisible à l'université : le cas des antennes universitaires ", Sociologie du travail, $\mathrm{n}^{\circ} 46$.

Maillard D., Veneau P. (2006), «Les licences professionnelles. Formes et sens pluriels de la "professionnalisation" à l'université, Sociétés contemporaines, 2006/2, nº 62.

Maillard F. (dir.), (2012), Former, certifier, insérer. Effets et paradoxes de l'injonction à la professionnalisation, Rennes, Presses universitaires de Rennes.

Moreau G. (2003), Le Monde apprenti, Paris, La Dispute.

Musselin C. (2008), Les universitaires, Paris, La Découverte.

Noiriel G. (2008), Introduction à la socio-histoire, Paris, La Découverte.

Quenson E., Coursaget S. (2012), La professionnalisation de l'enseignement supérieur, Toulouse, Octarès.

Stavrou S. (2011), "La "professionnalisation” comme catégorie de réforme à l'université en France ", Cahiers de la recherche sur l'éducation et les savoirs [En ligne], Hors-série nº 3.

Tanguy L. (2002) « La mise en équivalence de la formation avec l'emploi dans les IVième et Vème Plans (1962-1970)", Revue Française de sociologie 43 (4).

Thin D. (2010), Milieux populaires et logiques socialisatrices dominantes: une analyse de la confrontation, mémoire d'Habilitation à Diriger des Recherches, Université Lyon.

Tralongo S. (2015a), « Devenir un professionnel de la professionnalisation des étudiants : un changement accompagné dans les cultures et pratiques des enseignants et enseignants-chercheurs?", Revue Education et Socialisation, Varia 38.

Tralongo S. (2015b), «Le "Carnet de bord” utilisé en module de professionnalisation en IUT : un outil de cadrage pour le "cheminement" des étudiants? ", Spirale, n 55.

Zolesio E (2012), "La chirurgie et sa matrice de socialisation professionnelle ", Sociologie, 4, Vol. 3. 
